\title{
Comparing Tag Clouds, Term Histograms, and Term Lists for Enhancing Personalized Web Search
}

\author{
Orland Hoeber and Hanze Liu \\ Department of Computer Science, Memorial University \\ St. John's, NL, Canada AlB 3X5 \\ \{hoeber, hl5458\}@mun.ca
}

\begin{abstract}
Although static ranked lists remain the dominant Web search interface, they can limit the ability of Web searchers to find desired information when it is buried deep in the collection of search results. Web search visualization and Web search personalization are two active research directions that have shown promise for improving the user experience while searching the Web. In this paper, we propose three methods for visually representing information available within a personalized Web search system: tag clouds, term histograms, and term lists. These approaches not only describe the personalization model to the searcher, but also support interactive re-ranking of the search results. While preliminary evaluations did not find improvements beyond what was achieved with the personalization system, measures of subjective reactions showed an increased satisfaction in the search results. These results indicate that visual and interactive methods can be valuable for providing searchers with a sense of awareness and control during the search process.
\end{abstract}

Keywords-web search; visualization; personalization; user evaluations

\section{INTRODUCTION}

As the amount of information on Web continues to grow, Web search has become an essential tool for people to find valuable information from among the billions documents available. While static ranked lists are useful interfaces when the underlying search engine can ensure that relevant documents are placed high in the list, they are less effective for difficult or ambiguous queries that produce search results that are a mix of relevant and non-relevant documents.

Ranked lists represent the search results in a textual format. Reading text is a serial processing activity, which is slow and involves a large amount of mental workload [1]. As such, finding useful information from a ranked list is both time-consuming and mentally taxing. Moreover, it is difficult to understand the reason for the ranked order. The ranked list only tells users that a document high in the list is better than one low in the list; the reason why it is better may not be obvious to the searcher.

Another common problem of most Web search systems is that they do not consider the differences between individual users. The outcome of the search process relies solely upon the query. The underlying search engine provides the same search results for different users when they input the same query, regardless of their different information needs and preferences.

Two promising areas of research that have been pursued to address these shortcomings are visualization and personalization [2]. Visualization approaches provide interactive graphical representations of information relevant to the search process, with the goal of enhancing the searcher's understanding of the underlying features of the search results, and promoting interactive exploration and refinement activities. Personalization approaches dynamically model the searcher's interests, providing a customized ranking of the search results based on past activities.

In this paper, we explore combining these two approaches to improve the Web search process. The core personalization framework in this research is miSearch, a simple yet effective Web search personalization system [3]. It is enhanced with three different approaches to visually representing the personalization model: tag clouds, term histograms, and term lists. Each of these approaches allow the searcher to both see the reason for the personalized ranking of the search results, and interactively re-rank the search results based on terms they feel are important to their information need.

The remainder of this paper is organized as follows. Previous work related to Web search personalization and Web search visualization is outlined in Section 2. Details on the three visual enhancements to miSearch are explained in Section 3. The methods and results of a preliminary user evaluation are provided in Section 4. The paper concludes with a summary of what we have learned, and an overview of future research directions.

\section{RELATED WORK}

A number of different approaches to Web search personalization have been proposed in the literature. While the goal of providing a personalized ranking of the search results remains the same, the method by which a mathematical model of a searcher's interests is generated can be very different. Some allow searchers to generate explicit topics and select what information should be used to generate the personalization model [4]. An alternate approach is to generate a single model for each user, based on information found in documents on the searcher's computer system [5]. 
Others have explored methods for capturing both longterm and short-term interests based on the searcher's Web browsing activities, and filling in the gaps using information from similar users [6].

The core personalization system used in this research is miSearch [3]. It enables individual searchers to maintain multiple topics of interest, allowing them to choosing the appropriate one based on their current search needs. The personalization models are generated and updated automatically as the searcher clicks on documents from the search results list which they think are important. Evaluations have found the system to be very effective, even when selecting as few as two relevant documents from the search results list. The research question addressed in this paper is whether miSearch can be further enhanced using visual and interactive features.

Tag clouds provide a visual representation of a set of terms, wherein the terms are provided in alphabetical order and visual features such as size, weight, and colour are used to encode attributes of the associated terms [7]. Within the context of Web search, tag clouds have been used to provide a visual summary of search results [8], to summarize a collection of documents, showing the differences between individual documents and the whole collection [9], and to describe interest models in Web search personalization [4].

Term histograms are an alternate approach to visually encoding information associated with a collection of terms. Terms are listed in the order of the key attribute being represented, and a graphical bar is used to encode this value. They have been used to visually encode the frequency of term occurrence within search result sets [10].

Term lists are perhaps the simplest way to show information about a collection of terms. They are generally listed alphabetically, with no additional information provided other than the existence of a particular term within the collection. Term lists have been explored extensively within the context of interactive query expansion, with mixed results [11]-[13].

\section{Visual Enhancements to Personalized Web SEARCH}

Three different visual enhancements to the miSearch Web search personalization system have been implemented using the same source of information: the vector-based topic profiles used by miSearch to produce a personalized reranking of the search results [3]. These topic profiles are automatically generated by learning the searchers' interests in explicitly defined search topics. As searchers click on documents they believe are relevant, the frequency of the terms within the title, snippet, and URL are added to the topic profile vectors. While stemming is used in this process, the most frequent variant is tracked and used in the presentation of the terms in each of the visual enhancements described below.

\section{A. Tag Clouds}

Within the tag clouds, terms are organized in alphabetical order and presented in a compact space. The font size of each term is used to visually encode the relative vector weight in the topic profile. Since the range of values in the vector can be very broad, font size scaling is applied to avoid the situation where the fonts of high-value terms dwarf those of low-value terms. This feature is implemented using a log function, allowing the differences in the font sizes to still be perceived and decoded, even when there is a large difference between the highest and lowest values.

A screenshot of the tag cloud representation is shown in Figure 1(a). The red terms in the tag clouds are those that are present in the query. By clicking on these red terms, a searcher can re-rank the search results based on the separate keywords from the query. The other terms in the tag cloud are those that exist within the topic profile. These terms may also be used to re-rank the search results in a similar manner. Multiple terms can be selected, producing a weighted reranking of the search results. Any term selected for reranking is indicated with a box surrounding the selected term; de-selection is supported in the same manner as selection.

\section{B. Term Histograms}

Figure 1(b) demonstrates the term histogram approach. The same information from topic profiles are used, but in a different layout. The terms in the term histogram are arranged vertically, in order of the term weight in the topic profile vector. A graphical bar is provided with each term, such that the length of the bar encodes the weight of the associated term. In this way, the high weight terms are listed on the top, and indicated as such using the longest bar. The order of terms and the lengths of the bars allow the searcher to intuitively perceive the relative weight of the terms in the topic profile and the relative differences between specific terms.

As with the tag clouds, the terms from the query are labeled using a red colour, and the selected terms are marked using enclosing boxes. The same interactive features are provided, allowing the searcher to select or unselect terms for re-ranking the search results.

\section{Term Lists}

The term list is the simplest visual enhancement among the three, as represented in Figure 1(c). Term lists are implemented as simple vertical lists of the terms, provided in alphabetical order. No other information regarding the terms are provided. As such, the vector weights which are used as the main information visually encoded in the tag clouds and term histograms, is absent in term list. Therefore, term list does not provide any information other than the existence of a particular term in the topic profile. 


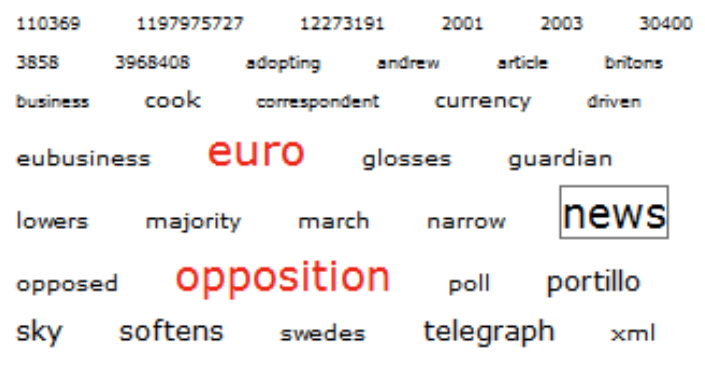

(a) Tag cloud

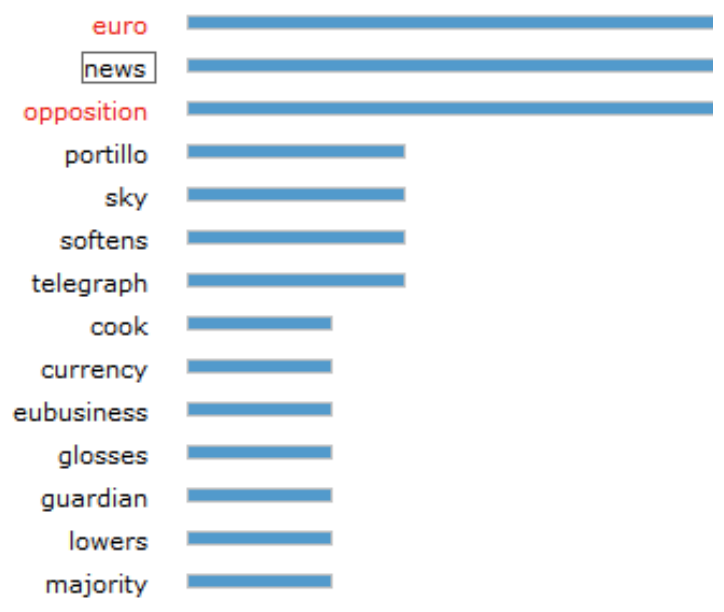

(b) Term histogram

correspondent
currency
driven
eubusiness
euro
glosses
guardian
lowers
majority
march
narrow
news
opposed
opposition

(c) Term list

Figure 1. Three approaches to the visual representation of a personalization model. Note that the red font indicates terms that are in the query, and the box enclosing a term indicates that it has been selected for re-ranking the search results.

Although the term list encodes no quantitative information for differentiating the terms, it still provides the same interactive features as the tag clouds and term histograms. The search results can be re-ranked by clicking on one or more terms in the list. Also, the same red colour encoding is used to indicate terms that are present in the query, and the same enclosing boxes mark the selected terms.

\section{EVALUATION}

The primary objective of the evaluation reported in this paper is to determine the usefulness and subjective satisfaction of adding visual representations of a searcher's interest in specific topics to a Web search personalization framework. The methods of the study and the results are provided below.

\section{A. Methods}

A set of 12 queries and brief descriptions of the information need were selected from the TREC 2005 Hard Track $^{1}$ test collection for their ambiguity. For each of these queries, the search results were pre-fetched from Yahoo and assigned a relevance score by a panel of three knowledgeable searchers. Using each of the three visual extensions of miSearch, the participants selected all of the terms that they felt were relevant to the query. The system automatically calculated the precision (ratio of relevant documents to the set of the top 10 and 20 documents) based on the reranking that was achieved by the participants' selections. Time to task completion and subjective reactions regarding each method were also be measured.

The study was designed as a $12 \times 3$ (search task $\mathrm{x}$ interface) mixed design. The search tasks were assigned betweensubjects to minimize learning effects; the interface was assigned within-subjects to support direct comparisons. The order of search task assignment was varied using a Latin Square approach. The interface exposure occurred in a round-robin fashion. As a result, each participant ended up using each of the three interfaces to conduct four different searches.

Preliminary results from this study include data from six participants, resulting in a total of 24 searches for each of the three alternate methods for visually enhancing the Web search personalization system. Although there is not sufficient data to perform reliable statistical analysis, trends within the data have been identified and are discussed below.

\section{B. Results}

With respect to the ability of participants to improve upon the precision scores achieved by the underlying personalization system, the results were mixed. Some participants were able to use some of the visual enhancements to achieve an increased precision among the top 10 and 20 documents for some search tasks. The choices made by other participants resulted in a decrease in precision. On average, there was negligible change in the precision scores, regardless of whether the tag cloud, term histogram, or term list was used.

In general, there was very little ability to improve upon the order of the search results beyond what the personalization system was already doing. This shouldn't necessarily be

\footnotetext{
${ }^{1}$ http://trec.nist.gov/data/t14_hard.html
} 
considered a negative result, given that the personalization system was already able to provide significant improvements over what the underlying search engine provided [3]. Further, the lack of difference between the three different approaches studied may be attributed to the fact that they all used the same source of information, and all were equally effective in allowing the searcher to interactively re-rank the search results.

In terms of the time taken to evaluate and make selections for re-ranking the search results, on average participants were fastest using the term histogram (94 seconds), followed by the tag cloud (116 seconds), and the term list (124 seconds). However, this data was highly variable, and depended to a great degree upon the skill of the participant and their familiarity with the search tasks.

If we are unable to improve upon the performance of the underlying personalized search system, what is the value of adding such visual and interactive enhancements? Beyond allowing the searcher to interact with the tag cloud, term histogram, and term list, these approaches all provided additional information to the searcher regarding the personalization model that was used to automatically re-rank the search results. Further, when used interactively, they provided the searcher with a sense of control over the reranking process. As such, measures of satisfaction with the search results and interface preference are important.

For each search task, participants were asked to report their satisfaction with the search results using a five-point Likert scale, before and after using the assigned interface. This data was analyzed to determine whether the participant's satisfaction changed as a result of having used the interface. The results of this analysis are reported in Figure 2. A somewhat surprising outcome is that participants reported an increase in satisfaction more frequently after having used the simpler and less visual approaches (e.g., term list and term histogram). The tag cloud resulted in the least number of increases in the satisfaction of the search results, providing evidence that it may be difficult to decode and use effectively.

Regardless of type of visual approach used to enhance the personalization system, $63 \%$ of the time participants reported an increase in satisfaction with the search results. In only $6 \%$ of the searches did the participants report a decrease. This is an important outcome given that the precision of the search results did not change noticeably. It provides a strong rationale for presenting the searcher with some kind of visual representation of the information used to re-rank the search results and allowing them to have some control over this process.

At the end of the study, participants were asked to rank which interface they preferred to use. These results are reported in Figure 3. Although this ranking was somewhat mixed, the term histogram was ranked number one most frequently, followed closely by the term list. The tag cloud

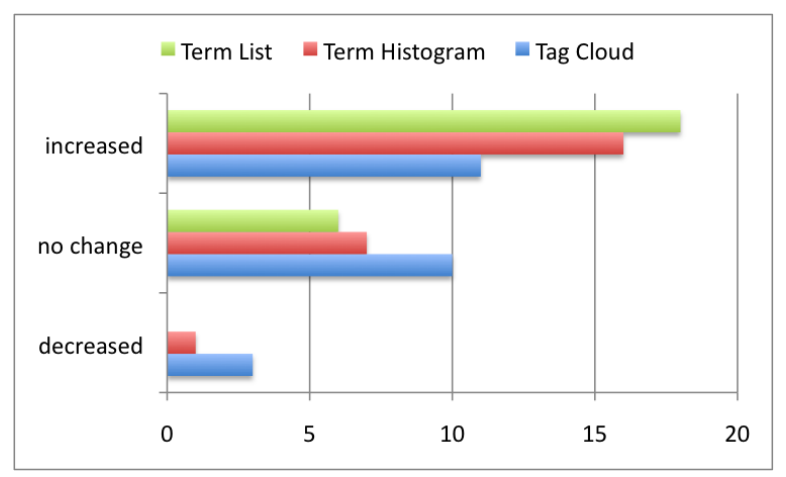

Figure 2. Changes in satisfaction with the search results after using the three alternate approaches for presenting and interacting with information present in the personalization model.

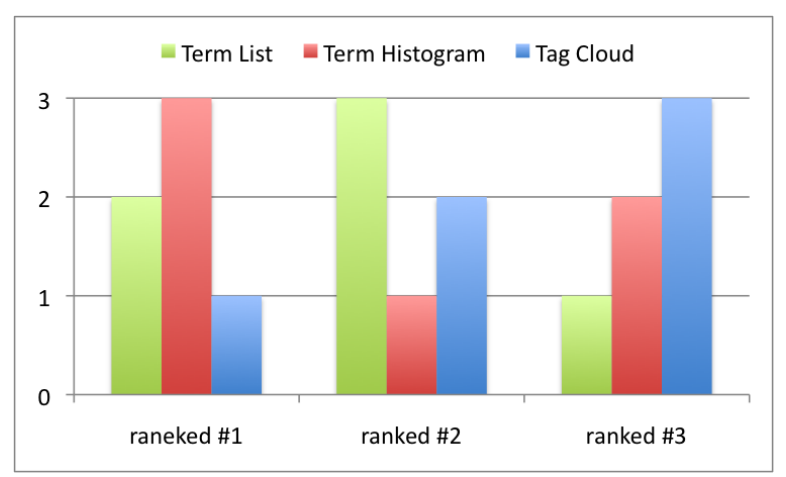

Figure 3. Preference rank for each of the visual enhancements studied.

was the preferred interface for only one participant. This adds further evidence in favour of using visual approaches that are easy to understand and decode for enhancing personalization systems.

\section{CONCLUSiOns \& Future Work}

The goal of this paper was to evaluate three different approaches for enhancing the miSearch Web search personalization system. Tag clouds, term histograms, and term lists were generated based on information in the personalization model. Participants used these visual and interactive approaches to re-rank the search results. While in some cases the precision among the top 10 and 20 search results increased, in other cases it decreased. On average, there was virtually no change in the precision of the search results after using the three different methods.

However, in nearly two-thirds of the cases, the participants reported an increase in the satisfaction with the search results set. This result indicates that there is value in proving the searcher with more awareness and control over what leads to the ranking of the search results. Although this outcome was found in the context of enhancing personalized Web search, it is inline with previous findings in interactive search results exploration [14]. 
Contrary to our expectations, participants in this study did not prefer the tag cloud approach to representing information from the personalization model. We had hypothesized that the compact representation and subtle visual encoding would have been well-received. Instead, the participants reported a more frequent increase in satisfaction, and a higher preference rank for the term histogram and term list methods. It appears that these simpler approaches are easier to decode, interpret, and use in this context.

We are currently in the process of quadrupling the number of participants in the study. This will allow us to analyze the results on a per-task basis to further understand the conditions under which improvements can be achieved. Further study of the reasons searchers might prefer one method to another is also warranted. A longitudinal study of these approaches under real-world search conditions will provide further insight into the value of the approaches for people conducting their regular search tasks and generating their own personalization models.

\section{ACKNOWLEDGEMENTS}

This research has been made possible the first author's Discovery Grant provided by the Natural Science and Engineering Research Council of Canada (NSERC).

\section{REFERENCES}

[1] J. A. Wise, J. J. Thomas, K. Pennock, D. Lantrip, M. Pottier, A. Schur, and V. Crow, "Visualizing the non-visual: Spatial analysis and interaction with information from text documents," in Proceedings of IEEE Symposium on Information Visualization, 1995, pp. 51-58.

[2] O. Hoeber, "Web information retrieval support systems: The future of web search," in Proceedings of the IEEE/WIC/ACM International Conference on Web Intelligence - Workshops (International Workshop on Web Information Retrieval Support Systems), 2008, pp. 29-32.

[3] O. Hoeber and C. Massie, "Automatic topic learning for personalizaed re-ordering of web search results," in Proceedings of the Atlantic Web Intelligence Conference, 2009, pp. 105116.

[4] J. Ahn, P. Brusiloviksy, D. He, J. Grady, and Q. Li, "Personalized Web exploration with task models," in Proceedings of the World Wide Web Conference, 2008, pp. 1-10.
[5] Z. Ma, G. Pant, and O. R. L. Sheng, "Interest-based personalized search," ACM Transactions on Information Systems, vol. 25, no. 1, pp. 1-38, 2007.

[6] K. Sugiyama, K. Hatano, and M. Yoshikawa, "Adaptive Web search based on user profile construction without any effort from users," in Proceedings of the World Wide Web Conference, 2004, pp. 675-684.

[7] A. W. Rivadeneira, D. M. Gruen, M. J. Muller, and D. R. Millen, "Getting our head in the clouds: toward evaluation studies of tagclouds," in Proceedings of the SIGCHI Conference on Human Factors in Computing Systems, 2007, pp. 995-998.

[8] B. Y.-L. Kuo, T. Hentrich, B. M. Good, and M. D. Wilkinson, "Tag clouds for summarizing Web search results," in Proceedings of the World Wide Web Conference, 2007, pp. 1203-1204.

[9] G. Xexeo, F. Morgado, and P. Fiuza, "Differential tag clouds: Highlighting particular features in documents," in Proceedings of the IEEE/WIC/ACM International Conference on Web Intelligence - Workshops (International Workshop on Web Information Retrieval Support Systems), 2009, pp. 129-132.

[10] O. Hoeber and X. D. Yang, "Interactive Web information retrieval using WordBars," in Proceedings of the IEEE/WIC/ACM International Conference on Web Intelligence, 2006, pp. 875-882.

[11] D. Harman, "Towards interactive query expansion," in Proceedings of the ACM SIGIR Conference on Research and Development in Information Retrieval, 1988, pp. 321-331.

[12] M. Magennis and C. J. van Rijsbergen, "The potential and actual effectiveness of interactive query expansion," in Proceedings of the ACM SIGIR Conference on Research and Development in Information Retrieval, 1997, pp. 324-332.

[13] I. Ruthven, "Re-examining the potential effectiveness of interactive query expansion," in Proceedings of the ACM SIGIR Conference on Research and Development in Information Retrieval, 2003, pp. 213-220.

[14] O. Hoeber and X. D. Yang, "Evaluating WordBars in exploratory Web search scenarios," Information Processing and Management, vol. 44, no. 2, pp. 485-510, 2008. 